\title{
Hermite function solutions of the Schrödinger equation for the sextic oscillator
}

\author{
A.M. Ishkhanyan ${ }^{1,2}$ \\ 1 Russian-Armenian University, Yerevan, 0051 Armenia \\ ${ }^{2}$ Institute for Physical Research, NAS of Armenia, Ashtarak, 0203 Armenia \\ G. Lévai \\ Institute for Nuclear Research, (Atomki), \\ H-4001 Debrecen, P.O. Box 51. Hungary
}

\begin{abstract}
We examine the conditions under which the solution of the radial stationary Schrödinger equation for the sextic anharmonic oscillator can be expanded in terms of Hermite functions. We find that this is possible for an infinite hierarchy of potentials discriminated by the parameter setting the strength of the centrifugal barrier. The $N$ 'th member of the hierarchy involves $N$ solutions for $N$ generally different values of the energy. For a particular member of the hierarchy, there exist infinitely many bound states with square integrable wave functions, written in terms of the Hermite functions, which vanish at the origin and at infinity. These bound states correspond to distinct values of the parameter setting the strength of the harmonic term. We also investigate connection with the polynomial solutions of the sextic oscillator obtained from the formalism of quasi-exactly solvable potentials.
\end{abstract}

PACS numbers: 03.65.Ge Solutions of wave equations: bound states, 02.30.Ik Integrable systems, 02.30.Gp Special functions

Keywords: Schrödinger equation; sextic oscillator; bi-confluent Heun equation; quasi-exactly solvable potential 


\section{INTRODUCTION}

The search for new exactly solvable quantum mechanical models is as old as quantum mechanics itself. In the beginning, these models were developed as illustrations to the mathematical and conceptual novelties brought about by the new theory. Later they proved to be important tools to construct bases for the description of realistic quantum mechanical systems, so their practical use was also demonstrated in addition to their internal beauty. These two aspects seem to maintain interest in exactly solvable models, and in general, in integrable systems even after the widespread use of numerical and computational techniques. They are still found useful in situations, when extreme accuracy is needed, e.g. in the description of symmetries, transitions through critical phases, or highly excited states.

Exact solvability of a quantum mechanical problem is usually understood as the requirement to give closed formulas for the energy eigenvalues and wave functions for bound states, as well as for the quantities related to scattering (if applicable). Generally the potential is expected to be energy-independent, although this is not a strict requirement. The solutions of the Schrödinger equation are usually expressed in terms of some special functions of mathematical physics that satisfy a second-order differential equation. In the first and simplest examples (e.g. the harmonic oscillator, Coulomb problem, etc.) these were found to be the classical orthogonal polynomials (for bound states) and the hypergeometric and confluent hypergeometric functions (for general solutions). These functions were wellknown mathematically even before the introduction of quantum mechanics, so the common mathematical knowledge accumulated about them could be easily applied to derive exactly solvable quantum mechanical problems. The potentials solvable in terms of these functions, the Natanzon-class potentials [1] have been discussed systematically and are generally well-understood [2, 3], see also Chapter 7 of Ref. [4].

However, there are potentials for which the framework based on the (confluent) hypergeometric functions is not adequate. There are well-known examples solved in terms of Bessel functions (e.g. the finite spherical square well or the exponential potential), or polynomials beyond the class of classical orthogonal plynomials. An example for these latter types are the quasi-exactly solvable (QES) potentials [5]. These potentials typically support infinite number of bound states, but closed solutions can be given only for the lowest few of them. The solutions are written in terms of a power series expansion, with coefficients satisfying 
a three-term recurrence relation. This expansion can be terminated by a specific choice of the potential parameters, leading to a polynomial form that describes the lowest few energy levels.

Another approach generalizing the range of exactly solvable potentials is the application of more general special functions satisfying a second-order differential equation. A recent attempt is considering various versions of the Heun differential equation [6] 8] and transforming them into te Schrödinger equation using the usual techniques applied for the Natanzon potentials. An advantage of these problems is that they allow more parameters and thus allow potentials with more general structure [3, 9-14]. Furthermore, since the Heun-type differential equations contain that of the hypergeometric and confluent hypergeometric functions, the potentials constucted in this way contain the Natanzon-class potentials as special cases. However, there is a serious problem: the solutions of the Heun-type differential equations are much less well-known than the (confluent) hypergeometric functions, so the construction of the wave functions is often highly non-trivial. The situation is, thus rather different from that experienced in the past, when the ready-made mathematical results could be used to develop exactly solvable quantum mechanical models. One possibility is expanding the solutions in terms of known special functions [14 23]. Solutions of the bi-confluent Heun equation (BHE) expanded in terms of Hermite functions have been considered Ref. [14].

A further interesting aspect of exactly solvable potentials is that sometimes their description is possible in terms of rather different approaches. The sextic oscillator, for example, has been discussed first as a QES potential [5], but it also appeared as a potential that can be derived from the bi-confluent Heun equation [24]. Various aspects of quasi-exact solvability have been discussed for the sextic oscillator [25 28, and its application in nuclear physics to describe various phase transitions has also been proposed [29 31]. The first results from the BHE approach concerned the analysis of the reduced sextic oscillator as a radial problem, i.e. the case when the quartic term was missing. It was shown that in this case the Hermite functions used in the expansion reduce to Hermite polynomials, and the solutions obtained from the QES and BHE approaches can be matched exactly.

The purpose of the pesent work is to extend these studies to the general form of the sextic oscillator, i.e. that containing also the quartic term. There are several questions arising naturally. First, under which conditions can the solutions be written in terms of Hermite functions? Second, how these solutions reduce to the polynomial form appearing in 
the QES discussion of the general form of the sextic oscillator? It is hoped that the answer to these questions will help the efforts of constructing solutions of the Heun-type differential equations in terms of expansions of other special functions.

The paper is arranged as follows. In Section II the solutions of the general sextic oscillator are presented in terms of Hermite functions. The discussion of these solutions is given in Section III, together with their connection with the solutions obtained from the QES ap-

proach. Particular examples are then presented in Section IV for the general non-polynomial solutions, while in Section $\mathrm{V}$ the discussion of the results is given.

\section{SOLUTIONS IN TERMS OF THE HERMITE FUNCTIONS}

Here we consider the one-dimensional stationary Schrödinger equation for a particle of mass $m$ and energy $E$

$$
\frac{\mathrm{d}^{2} \psi}{\mathrm{d} r^{2}}+\frac{2 m}{\hbar^{2}}(E-V(r)) \psi=0
$$

with $r \in[0, \infty)$. This problem can be obtained from a spherically symmetric potential after the separation of the angular variables. In this case the centrifugal term appears, which can be unified with any $r^{-2}$-like term that may appear. The Schrödinger equation defined in this way for the sextic oscillator potential

$$
V(r)=\frac{V_{-2}}{r^{2}}+V_{0}+V_{2} r^{2}+V_{4} r^{4}+V_{6} r^{6}
$$

can be transformed into the bi-confluent Heun equation [ 6 [ 8 ]

$$
\frac{\mathrm{d}^{2} u}{\mathrm{~d} z^{2}}+\left(\frac{\gamma}{z}+\delta+\varepsilon z\right) \frac{\mathrm{d} u}{\mathrm{~d} z}+\frac{\alpha z-q}{z} u=0,
$$

via the change of the variables [3, 9, 14]

$$
\psi(r)=z^{\alpha_{0}} e^{\alpha_{1} z+\alpha_{2} z^{2}} u(z), z=r^{2} / 4
$$

The involved parameters are given by the equations (see Ref. [14])

$$
\begin{gathered}
\gamma=1 \pm \frac{1}{2}\left(1+\frac{8 m V_{-2}}{\hbar^{2}}\right)^{1 / 2}, \quad \delta=\frac{64 m V_{4}}{\hbar^{2} \varepsilon}, \quad \varepsilon= \pm 16\left(\frac{2 m V_{6}}{\hbar^{2}}\right)^{1 / 2} \\
\alpha=-\frac{8 m V_{2}}{\hbar^{2}}+\frac{\delta^{2}}{4}+\frac{\gamma+1}{2} \varepsilon, \quad q=-\frac{\gamma \delta}{2}-\frac{2 m\left(E-V_{0}\right)}{\hbar^{2}}
\end{gathered}
$$


and

$$
\left(\alpha_{0}, \alpha_{1}, \alpha_{2}\right)=\left(\frac{2 \gamma-1}{4}, \frac{\delta}{2}, \frac{\varepsilon}{4}\right) .
$$

We note that here any combination of signs plus or minus for $\gamma$ and $\varepsilon$ is applicable. Notably, different combinations suggest different fundamental solutions. This observation can be used to construct the general solution of the problem.

For a non-zero $\varepsilon$, that is for $V_{6} \neq 0$, the solution of the bi-confluent Heun equation (3) allows series expansion in terms of the Hermite functions of a shifted and scaled argument [14]:

$$
u=\sum_{n=0}^{\infty} c_{n} H_{\nu_{0}+n}(\xi)
$$

where

$$
\xi= \pm(-\varepsilon / 2)^{1 / 2}(z+\delta / \varepsilon)
$$

The expansion coefficients $c_{n}$ obey a three-term recurrence relation and the index parameter $\nu_{0}$ may adopt two values: $\nu_{0}=0$ or $\nu_{0}=\gamma-\alpha / \varepsilon$. With $\nu_{0}=0$ the expansion functions become Hermite polynomials, while the second choice applies generally non-polynomial Hermite functions [8]. The $\nu_{0}=0$ case will be discussed briefly towards the end of Section III. Here we explore the non-polynomial expansion with $\nu_{0}=\gamma-\alpha / \varepsilon$. In this case the three-term recurrence relation obeyed by the expansion coefficients read [14]

$$
R_{n} c_{n}+Q_{n-1} c_{n-1}+P_{n-2} c_{n-2}=0
$$

with

$$
\begin{gathered}
R_{n}=\left(\frac{2}{-\varepsilon}\right)^{1 / 2} n[-\alpha+(\gamma+n) \varepsilon], \\
Q_{n}=\mp[q+(\gamma+n) \delta] \\
P_{n}=\frac{(\gamma+n) \varepsilon}{(-2 \varepsilon)^{1 / 2}}
\end{gathered}
$$

where the signs $\mp$ in the equation for $Q_{n}$ refer to the choices \pm for the argument $\xi$.

This expansion terminates thus reducing to a closed-form solution involving a finite number of the Hermite functions if $\gamma$ is zero or a negative integer: $\gamma=-N, N=0,1,2, \ldots$, and the accessory parameter $q$ satisfies a polynomial equation of the degree $N+1$. Using the recurrence relation $H_{n}=2 z H_{n-1}-2(n-1) H_{n-2}$, any such a finite-term solution can be reduced to a linear combination of only two Hermite functions. As such functions, one may 
choose the contiguous functions $H_{-\alpha / \varepsilon}$ and $H_{-1-\alpha / \varepsilon}$ :

$$
u(z)=P_{0}(z) H_{-\alpha / \varepsilon}(\xi)+P_{1}(z) H_{-1-\alpha / \varepsilon}(\xi)
$$

The mentioned recurrence relation for the Hermite functions indicates that the coefficients of this combination are polynomials in $z$. It is readily understood that, for a given $N \geq 2, P_{0}(z)$ is of the degree $N-2$ and $P_{1}(z)$ is of the degree $N-1$. With the notation $s_{0}= \pm(-\varepsilon / 2)^{1 / 2}$, the explicit solutions for $N=0,1,2,3$ read

$$
\begin{gathered}
N=0: \gamma=0 \\
q=0 \\
u=H_{-\alpha / \varepsilon}(\xi) \\
N=1: \gamma=-1, \\
q^{2}-\delta q+\alpha=0 . \\
u=-s_{0}(q-\delta) H_{-\alpha / \varepsilon}(\xi)+\alpha H_{-1-\alpha / \varepsilon}(\xi), \\
N=2: \gamma=-2, \\
q^{3}-3 \delta q^{2}+2\left(\delta^{2}+\varepsilon+2 \alpha\right) q-4 \alpha \delta=0 . \\
u_{-3}\left(q^{2}-3 q \delta+2 \delta^{2}+2 \varepsilon\right) H_{-\alpha / \varepsilon}(\xi)+2 \alpha(q-\delta+\varepsilon z) H_{-1-\alpha / \varepsilon}(\xi), \\
q^{4}-6 q^{3} \delta+q^{2}\left(10 \alpha+11 \delta^{2}+10 \varepsilon\right)-6 q \delta\left(5 \alpha+\delta^{2}+3 \varepsilon\right)+9 \alpha\left(\alpha+2 \delta^{2}+2 \varepsilon\right)=0 . \\
u=-s_{0}\left[(q-3 \delta)\left(q^{2}-3 q \delta+2 \delta^{2}+10 \varepsilon+\alpha\right)+6 \varepsilon(2 \delta-\alpha z)\right] H_{-\alpha / \varepsilon}(\xi)+ \\
3 \alpha\left[\left(q^{2}-3 q \delta+2 \delta^{2}+4 \varepsilon+\alpha\right)+2(q-\delta) \varepsilon z+2 \varepsilon^{2} z^{2}\right] H_{-1-\alpha / \varepsilon}(\xi) .
\end{gathered}
$$

For definiteness, below we choose $s_{0}=(-\varepsilon / 2)^{1 / 2}$.

It is worthwhile to examine the structure of the solutions (8) in terms of the powers of $z$. For this we consider the formula

$$
H_{\nu}(y)=\sum_{k=0}^{\infty} \frac{2^{k}}{k !}\left(\begin{array}{l}
\nu \\
k
\end{array}\right) H_{\nu-k}\left(y_{0}\right)\left(y-y_{0}\right)^{k}
$$

with

$$
y=s_{0} z+s_{0} \frac{\delta}{\epsilon} \quad y_{0}=s_{0} \frac{\delta}{\epsilon} \quad \nu=\nu_{0}+n
$$


With these substitutions and some rearrangement we get

$$
u(z)=\sum_{k=0}^{\infty} \frac{2^{k}}{k !}\left(s_{0} z\right)^{k} \sum_{n=0}^{N} c_{n}\left(\begin{array}{c}
-\frac{\alpha}{\epsilon}-N+n \\
k
\end{array}\right) H_{-\frac{\alpha}{\epsilon}-N+n-k}\left(s_{0} \frac{\delta}{\epsilon}\right)
$$

It can be seen that the first few terms of this expansion vanish. The coefficient of $z^{0}$ is found to be nothig but $u(0)$ (see Eqs. (8) and (9)). If $\alpha_{0} \leq 0$, i.e. $\gamma \leq 1 / 2$ (as is the case now, due to $\gamma=-N)$, then the boundary conditon $\psi(0)=0$ prescribes $u(0)=0$, so in this case the coefficient of $z^{0}$ will be zero. For $k=1$ one obtains the expression

$$
2 s_{0} \frac{\delta}{\epsilon} \sum_{n=0}^{N} c_{n}\left(-\frac{\alpha}{\epsilon}-N+n\right) H_{-\frac{\alpha}{\epsilon}-N+n-1}\left(s_{0} \frac{\delta}{\epsilon}\right),
$$

which can be rewritten into a two-term relation using

$$
2 \nu H_{\nu-1}(z)=2 z H_{\nu}(z)-H_{\nu+1}(z)
$$

with $\nu=-\frac{\alpha}{\epsilon}-N+n$ and $z=s_{0} \delta / \epsilon$. Then the first term recovers the expression found for $k=0$, so it vanishes, and what remains is

$$
-s_{0} \frac{\delta}{\epsilon} \sum_{n=0}^{N} c_{n} H_{-\frac{\alpha}{\epsilon}-N+n+1}\left(s_{0} \frac{\delta}{\epsilon}\right) .
$$

It turns out that this expression is nothing but the algebraic condition prescribed for $q$, which comes from the requirement of the termination of the series. In particular, it turns into Eqs. (19), 22) and (25) for $N=1,2$ and 3, respectively. Actually, it can be seen that

all the coefficients of $z^{k}$ are zero, up to $k=N$, so $u(z)$ behaves like $z^{N+1}$ times a power series.

\section{GENERAL DISCUSSION OF THE SOLUTIONS}

We now apply these solutions to the sextic oscillator problem with the parameters of the corresponding bi-confluent Heun equation given by equations (5), (6). Four general observations are appropriate here.

(i) We first note that the parameter $\gamma$ depends only on the strength $V_{-2}$ of the centrifugalbarrier term. Then, choosing the minus sign in the first equation (5), the equation $\gamma=-N$, $N=0,1,2, \ldots$, results in

$$
V_{-2}=\frac{\hbar^{2}}{2 m}\left(\gamma-\frac{1}{2}\right)\left(\gamma-\frac{3}{2}\right)=\frac{\hbar^{2}(2 N+1)(2 N+3)}{8 m} .
$$


In explicit form, we have the sequence

$$
V_{-2}=\frac{3 \hbar^{2}}{8 m}, \frac{15 \hbar^{2}}{8 m}, \frac{35 \hbar^{2}}{8 m}, \frac{63 \hbar^{2}}{8 m}, \frac{99 \hbar^{2}}{8 m}, \frac{143 \hbar^{2}}{8 m}, \ldots, N=0,1,2,3,4,5, \ldots
$$

Thus, we see that the Hermite-function solutions compose an infinite countable set that can be numbered by an integer which is related to the value of the strength of the centrifugalbarrier term in potential (2).

(ii) Second, we observe that the accessory parameter $q$ is the only parameter that depends on the energy. It then follows that the second condition for a Hermite-function solution to exist, that is the $(N+1)$ 'th degree polynomial equation for $q$ (see equations (16), (19), 22) and (25) for $N=0,1,2,3$, respectively), since the dependence $q=q(E)$ is linear, presents a $(N+1)$ 'th degree polynomial equation for energy $E$. Hence, we see that, for a given set of fixed values of the potential parameters $V_{0,2,4,6}$ (without loss of the generality, one always may put $V_{0}=0$ ), the $N^{\prime}$ th member of the hierarchy of the Hermite-function solutions corresponds to $N+1$ fixed values of the energy.

(iii) Third, again examining the parameters (5), (6) of the bi-confluent Heun equation, we note that parameter $\delta$, which defines the shift in the argument $\xi$ of the involved Hermite functions (see equation (9) ), depends only on the strength $V_{4}$ of the quartic term of potential (2). Furthermore, for the reduced sextic oscillator, for which $V_{4}=0$ and thus the quartic term is absent in the potential, this parameter vanishes. We note that in this case some other parameters as well as the equations for the accessory parameter are rather simplified. In particular, in this case $q=-2 m\left(E-V_{0}\right) / \hbar^{2}$. Besides, it can be shown that the polynomial equations for $q$ are proportional to $q$ for all even orders $N=0,2,4, \ldots$ so that $q=0$ is a root for all of these equations. We then conclude that for the reduced sextic oscillator zero-energy $\left(E=V_{0}=0\right)$ Hermite-function solutions exist for all even orders. In fact, the zero energy solution for the reduced sextic oscillator for arbitrary value of the strength $V_{-2}$ of the centrifugal-barrier term (not only for those given by equation (33)) is written in terms of the confluent hypergeometric functions. This is because for $\delta=q=0$ the biconfluent Heun equation is exactly solved as [6]

$$
u=C_{1} \cdot{ }_{1} F_{1}\left(\frac{\alpha}{2 \varepsilon} ; \frac{\gamma+1}{2} ;-\frac{\varepsilon z^{2}}{2}\right)+C_{2} \cdot U\left(\frac{\alpha}{2 \varepsilon} ; \frac{\gamma+1}{2} ;-\frac{\varepsilon z^{2}}{2}\right)
$$

where $C_{1,2}$ are arbitrary constants and ${ }_{1} F_{1}$ and $U$ are the Kummer and Tricomi confluent hypergeometric functions, respectively. It is understood that this solution allows reduction 
to a combination of the Hermite functions if $\gamma$ is an integer so that $V_{-2}$ adopts the values given by equation (33). This is a useful observation that can be employed when discussing the bound states described by the Hermite-function solutions (see below).

(iv) Finally, fourth, the presented Hermite-function solutions may describe bound states, that is, the wave functions may be square integrable (in general they are not). Since $V_{-2}$ is positive for all $N=0,1,2,3, \ldots$ and, hence, for a positive $V_{6}$ potential (2) defines an infinite potential well tending to plus infinity for $x \rightarrow 0$ and $x \rightarrow+\infty$, the bound state wave functions should vanish both in the origin and at the infinity. Thus, one should consider the boundary conditions

$$
\psi(0)=0, \psi(+\infty)=0 .
$$

By examining the asymptotes of the involved Hermite functions, it is readily shown that the second of these conditions is satisfied if one chooses the minus sign for $\varepsilon$ :

$$
\varepsilon=-16\left(\frac{2 m V_{6}}{\hbar^{2}}\right)^{1 / 2}
$$

The boundary condition at the origin can be analyzed considering Eqs. (4) and (7), which imply $z^{\alpha_{0}}=z^{\gamma / 2-1 / 4}$. Taking into account also the requirement $\gamma=-N$ and that the $u(z)$ function behaves near the origin as $z^{N+1}$, wave function folows the pattern

$$
\psi(r) \sim z^{\frac{N}{2}+\frac{3}{4}} \sim r^{N+\frac{3}{2}}
$$

which is in accordance with the coefficient of the centrifugal term (33). Following the same arguments we obtain a transcendental equation of the form

$$
P_{0}(0) H_{-\alpha / \varepsilon}\left(s_{0} \delta / \varepsilon\right)+P_{1}(0) H_{-1-\alpha / \varepsilon}\left(s_{0} \delta / \varepsilon\right)=0 .
$$

Unless $\delta=0$, since $\alpha / \varepsilon$ in general is not an integer, this is a complicated equation the solution of which can be constructed only asymptotically. To do this, we note that, with the chosen negative $\varepsilon$ given by equation (37), the argument $s_{0} \delta / \varepsilon$ of the involved Hermite functions is real. Besides, examining the indexes of the Hermite functions, we observe that $\alpha / \varepsilon$ linearly depends on the strength $V_{2}$ of the harmonic term of the sextic oscillator potential (2). Hence, by considering sufficiently large $V_{2}$, one can achieve the condition $|y|<(2 v+1)^{1 / 2}$ the fulfillment of which indicates that a Hermite function $H_{\nu}(y)$ behaves oscillatory (see [8, 32]). Applying then the approximation [ㅇ, 32]

$$
H_{\nu}(y) \approx \frac{2 e^{y^{2} / 2} \Gamma(\nu)}{\left(1-\frac{y^{2}}{2 \nu+1}\right)^{1 / 4} \Gamma(\nu / 2)} \cos \left[\frac{\pi \nu}{2}-y\left(2 \nu-\frac{y^{2}}{3}+1\right)^{1 / 2}\right]
$$


one arrives at a rather accurate approximation in terms of elementary functions. More specific inspection shows that this is a rather accurate approximation for higher-order bound states or, alternatively, for relatively small values of the parameter $V_{4}$ (see the details below). The numerical testing supports this observation.

Before closing this Section, let us discuss the relation of the present formalism of he sextic oscillator with that based on the theory of quasi-exactly solvable potentials [5]. In this approach the potential is written in a form similar to that in Eq. (2) with

$$
\begin{aligned}
V_{-2} & =\left(2 s-\frac{1}{2}\right)\left(2 s-\frac{3}{2}\right), \\
V_{2} & =b^{2}-4 a\left(s+M+\frac{1}{2}\right), \\
V_{4} & =2 a b, \\
V_{6} & =a^{2} .
\end{aligned}
$$

The normalizable solutions are written as

$$
\psi(r)=C r^{2 s-1 / 2} \exp \left(-\frac{a r^{4}}{4}-\frac{b r^{2}}{2}\right) P_{M}\left(r^{2}\right),
$$

where normalizability requires $a>0$ ( $a=0$ recovers the radial harmonic oscillator problem) and the wave function vanishes at the origin for $s>1 / 4 . P_{M}\left(r^{2}\right)$ is a polynomial of the order $M$. Substituting the wave function (45) into the radial Schrödinger equation and separating the powers of $r^{2}$ one obtains a three-term recursion relation for the coefficients of the polynomial $P_{M}\left(r^{2}\right)$, represented by an infinite Jacoi-type matrix. With an appropriate choice of the parameters an off-diagonal matrix element can be set to zero, and thus an $(M+1)$-dimensional submatrix can be separated. In this way the polynomial coefficients of the first $M+1$ solutions can be obtained.

This QES methodology can be related to the method based on the expansion in terms of Hermite functions. Expressing the parameters of the latter method in terms of those of the former one, one finds

$$
\begin{aligned}
\gamma & =1+\Pi_{\gamma}|2 s-1| \\
\varepsilon & =16 a \Pi_{\varepsilon} \\
\delta & =4 b \Pi_{\varepsilon} \\
\alpha & =16 a\left(s+M+\frac{1}{2}+\Pi_{\varepsilon}+\Pi_{\varepsilon} \Pi_{\gamma}\left|s-\frac{1}{2}\right|\right) \\
q & =2 b \Pi_{\varepsilon}\left(1+\Pi_{\gamma}|2 s-1|\right)-\left(E-V_{0}\right)
\end{aligned}
$$


where $\Pi_{\varepsilon}$ and $\Pi_{\gamma}$ are the signs appearing in $\varepsilon$ and $\gamma$ in Eq. (7).

The QES wave functions correspond to the case when the Hermite functions reduce to Hermite polynomials in Eq. (8). As discussed previously, this is possible if $\nu_{0}$ is a nonnegative integer. In the simplest case $\nu_{0}=0$. Furthermore, due to the normalizability requirement, $\varepsilon<0$, i.e. $\Pi_{\varepsilon}=-1$ has to be taken. In this case $\gamma=2 s$ or $\gamma=2(1-s)$.

With the $\nu_{0}=0$ choice the recursion relations in Eqs. (11) to (13) are replaced by

$$
\begin{gathered}
R_{n}=\left(\frac{2}{-\varepsilon}\right)^{1 / 2} n[\alpha+(n-\gamma) \varepsilon], \\
Q_{n}=\mp\left[q+\delta\left(\frac{\alpha}{\epsilon}+n\right)\right], \\
P_{n}=\frac{\alpha+n \varepsilon}{(-2 \varepsilon)^{1 / 2}} .
\end{gathered}
$$

The condition for the termination of the recursion is now $\alpha / \varepsilon=-N$. With the $\Pi_{\gamma}=+1$ choce and taking $\gamma=2 s$ the parametrization of the sextic oscillator is recovered with the expected values of the $V_{i}$ coefficients (41) to (44), furthermore, the structure of wave functions is also reproduced with $M=N$. A special case of this problem has been obtained in our recent work [24]. There the reduced sextic oscillator (that without quartic term) was discussed by taking $\delta=0$. The $R_{n}, Q_{n}$ and $P_{n}$ coefficients found there agree with those in Eqs. (51) to (53) with $\delta=0$, and the equivalence of the solutions obtained from the QES and the present approaches was established.

The $\nu_{0}=\gamma-\alpha / \varepsilon$ case can also lead to an expansion in terms of Hermite polynomials. As discussed in the previous Section, in this case the $\gamma=-N$ choice has to be made with non-negative integer. Due to Eq. (46) this also implies $\Pi_{\gamma}=-1$ and $N=|2 s-1|-1$. For $s \geq 1 / 2$ we obtain $s=N / 2+1$ i.e. $s$ has to be an integer or half-integer exceeding $1 / 2$, which is in contrast with the case $\nu_{0}=0$. In summary, in this case $\nu_{0}+n=M+n+1$, i.e. the $z(z)$ function turns into a polynomial of the order of $M+N+1$. Recalling the arguments outlined a the end of Section 2, one finds that it is a polynomial of degree $M$ times $z^{N+1}$. Combining this with Eq. (38) and remembering that $s=N / 2+1$ the structure of the wave function (45) is recovered.

It is worth emphasizing the difference between the cases corresponding to $\nu_{0}=0$ and $\nu_{0}=\gamma-\alpha / \varepsilon$. In the latter case the solutions do not take a polynomial form, except when the parametrization is matched with that used in the QES approach. In this case the $\gamma$ variable is restricted to integer values, formally coresponding to half-integer values 
of the angular momentum. In the former case the solutions are always written in terms of polynomials, but there is no restrictin for the $\gamma$ variable except that prescribed by the normalizability near the origin for physical wave funtions.

\section{PARTICULAR EXAMPLES}

Consider the simplest case $N=0$ which differs from the rest in that it is the only case when the solution involves only one Hermite function. In this case $\gamma=0$ and $q=0$ so that $V_{-2}=3 \hbar^{2} /(8 m)$ and $E-V_{0}=0$. Putting $V_{0}=0$, the particular sextic oscillator potential reads

$$
V(r)=\frac{3 \hbar^{2}}{8 m r^{2}}+V_{2} r^{2}+V_{4} r^{4}+V_{6} r^{6}
$$

The zero-energy solution of the Schrödinger equation for this potential that vanishes at infinity (recall that we assume $V_{6}>0$ and take minus sign for both $\gamma$ and $\varepsilon$ ) explicitly reads

$$
\psi(r)=r^{-1 / 2} \exp \left[-\frac{m\left(V_{4}+V_{6} r^{2}\right) r^{2}}{2 \hbar\left(2 m V_{6}\right)^{1 / 2}}\right] H_{\nu}(\xi(r))
$$

where

$$
\xi(r)=\frac{V_{4}+2 V_{6} r^{2}}{2\left(2 \hbar^{2} V_{6}^{3} / m\right)^{1 / 4}}
$$

and

$$
\nu=\frac{V_{4}^{2}-4 V_{2} V_{6}}{8\left(2 \hbar^{2} V_{6}^{3} / m\right)^{1 / 2}}-\frac{1}{2}
$$

The bound-state wave functions should fulfill the condition

$$
H_{\nu}(\xi(0))=0
$$

This is an exact equation. In terms of dimensionless parameters

$$
\xi_{0}=\frac{V_{4}}{2\left(2 \hbar^{2} V_{6}^{3} / m\right)^{1 / 4}}, \quad w=\frac{V_{2}}{\left(2 \hbar^{2} V_{6} / m\right)^{1 / 2}}
$$

the equation is rewritten as

$$
H_{\left(\xi_{0}^{2}-w-1\right) / 2}\left(\xi_{0}\right)=0
$$

In the two-dimensional space of parameters $\left(\xi_{0}, w\right)$, this equation defines a countable infinite set of unbounded smooth curves (Figure 1). The curves do not cross. 


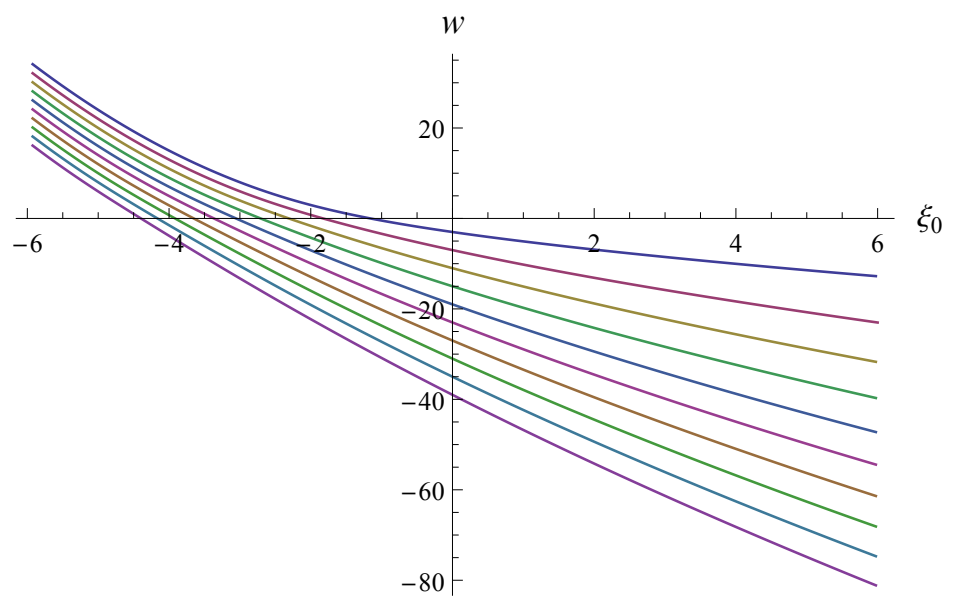

FIG. 1. The first ten of $\left(\xi_{0}, w\right)$ curves defined by equation 60$)(n=1, \ldots, 10)$.

For the reduced sextic oscillator for which $V_{4}=0$, we have $\xi_{0}=\xi(0)=0$. The Hermite function is then written in terms of the Euler gamma function as

$$
H_{\nu}(0)=\frac{\pi^{1 / 2} 2^{\nu}}{\Gamma\left(\frac{1-\nu}{2}\right)}
$$

Hence, we conclude that $\nu$ is a positive odd integer: $\nu=2 n-1, n=1,2,3, \ldots$. With this, we obtain the exact result

$$
V_{2}=(1-4 n)\left(\frac{2 \hbar^{2} V_{6}}{m}\right)^{1 / 2}, n=1,2,3, \ldots
$$

We note that in this case, since $\nu$ is a positive integer, the Hermite function reduces to a Hermite polynomial and, hence, the bound state wave functions become quasi-polynomials.

For a non-zero $V_{4}$, however, the wave function is not a quasi-polynomial. Using the approximation 40 , we have the equation

$$
\cos \left[\frac{\pi \nu}{2}-\xi_{0}\left(2 \nu-\frac{\xi_{0}^{2}}{3}+1\right)^{1 / 2}\right] \approx 0, \quad \nu=\frac{\xi_{0}^{2}-w-1}{2}
$$

from which we derive

$$
\frac{V_{2}}{\left(2 \hbar^{2} V_{6} / m\right)^{1 / 2}} \approx(1-4 n)+\xi_{0}^{2} \frac{\pi^{2}-8}{\pi^{2}}-\xi_{0} \frac{4}{\pi}\left[\frac{12-\pi^{2}}{3 \pi^{2}} \xi_{0}^{2}-(1-4 n)\right]^{1 / 2}
$$

$n=1,2,3, \ldots$ This is a rather accurate approximation if $\left|V_{4}\right| / V_{6}^{1 / 2}$ is less than or of the order of one. The accuracy improves with higher $n$. For instance, for $\left|V_{4}\right| / V_{6}^{1 / 2} \approx 1$ the 


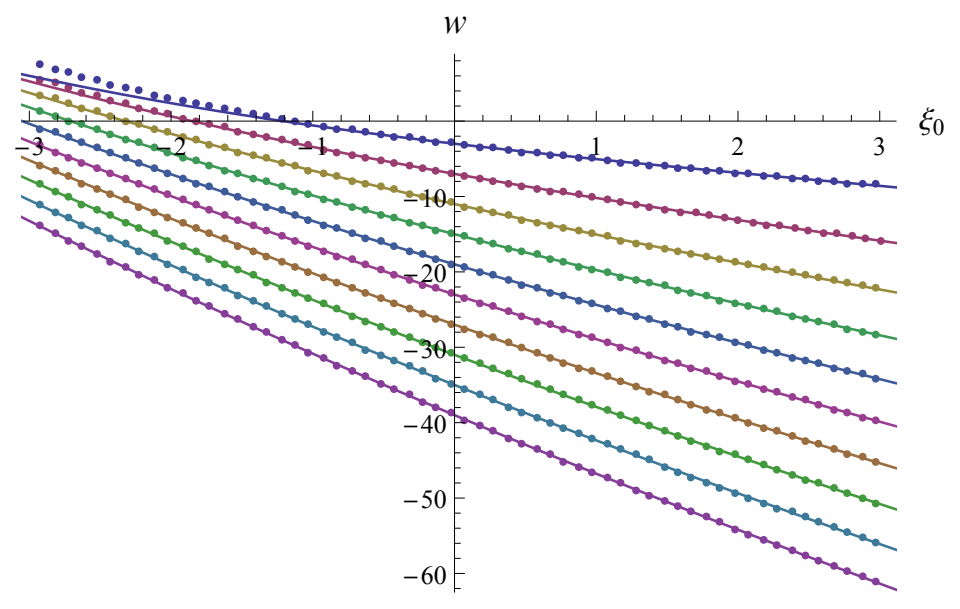

FIG. 2. Comparison of approximation (64) (solid curves) with the numerical solution of equation (60) (points).

relative error is of the order of $10^{-3}$ for $n=1$ and it becomes of the order of $10^{-5}$ for $n=7$. Comparison of this approximation with the exact numerical result is shown in Figure 2.

Consider now the case $N=1$. For this case $\gamma=-1$ and the potential is given as (we put $\left.V_{0}=0\right)$

$$
V(r)=\frac{15 \hbar^{2}}{8 m r^{2}}+V_{2} r^{2}+V_{4} r^{4}+V_{6} r^{6}
$$

The solution of the Schrödinger equation for this potential that vanishes at the infinity reads

$$
\begin{aligned}
\psi(r)= & r^{-3 / 2} \exp \left[-\frac{m\left(V_{4}+V_{6} r^{2}\right) r^{2}}{2 \hbar\left(2 m V_{6}\right)^{1 / 2}}\right] \\
& \times\left[\frac{2^{1 / 2} m / \hbar^{2}}{\left(2 m V_{6} / \hbar^{2}\right)^{1 / 4}}\left(E-\frac{V_{4}}{\left(2 m V_{6} / \hbar^{2}\right)^{1 / 2}}\right) H_{\nu}(\xi(r))+4 \nu H_{\nu-1}(\xi(r))\right],
\end{aligned}
$$

where $\xi(r)$ is the same as in the previous case (Eq. (56)), while the index $\nu$ is given as (compare with (57))

$$
\nu=\frac{V_{4}^{2}-4 V_{2} V_{6}}{8\left(2 \hbar^{2} V_{6}^{3} / m\right)^{1 / 2}}
$$

The accessory parameter $q$ should now satisfy the equation $q^{2}-\delta q+\alpha=0$, which is simplified to $m\left(E-V_{0}\right)^{2}-2 \hbar^{2} V_{2}=0$. Hence, in this case the energy is only related to the strength $V_{2}$ of the harmonic potential term:

$$
E-V_{0}= \pm\left(\frac{2 \hbar^{2} V_{2}}{m}\right)^{1 / 2}
$$


With this, in order the solution (66) to describe a bound state, one should require the wave function to vanish in the origin. We then arrive at the equation

$$
\left[-\xi_{0} \pm\left(\xi_{0}^{2}-2 \nu\right)^{1 / 2}\right] H_{\nu}\left(\xi_{0}\right)+2 \nu H_{\nu-1}\left(\xi_{0}\right)=0
$$

Compared with (60), this is a more complicated equation. In terms of parameters $\left(\xi_{0}, w\right)$ given by equations (59) the equation is rewritten as

$$
\left(\xi_{0} \mp w^{1 / 2}\right) H_{\frac{\xi_{0}^{2}-w}{2}}\left(\xi_{0}\right)-\left(\xi_{0}^{2}-w\right) H_{\frac{\xi_{0}^{2}-w}{2}-1}\left(\xi_{0}\right)=0 .
$$

This equation has the trivial solution $\left.w=\xi_{0}^{2} \Rightarrow \nu=0, V_{2}=V_{4}^{2} /\left(4 V_{6}\right)\right)$. However, the wave function produced by this solution is identically zero.

The non-trivial solutions of equation (70) essentially depend on the sign of the energy. For negative energies (minus sign in $(68)$ and plus sign in (70)), the solution is shown in Figure 3. All the curves lay in the second quadrant of the $\left(\xi_{0}, w\right)$ plane, that is always $\xi_{0}<0, w \geq 0$ so that $V_{4} \leq 0, V_{2} \geq 0$. An accurate approximation for these curves is given by the simple formula

$$
w \approx \xi_{0}^{2}-2 n, \quad n=1,2,3, \ldots
$$

This means that the eigenvalues are very close to those derived by polynomial reduction of equation (70). The absolute error of the approximation for the most unfavorable case $n=1$ is shown in the inset of Figure 3.

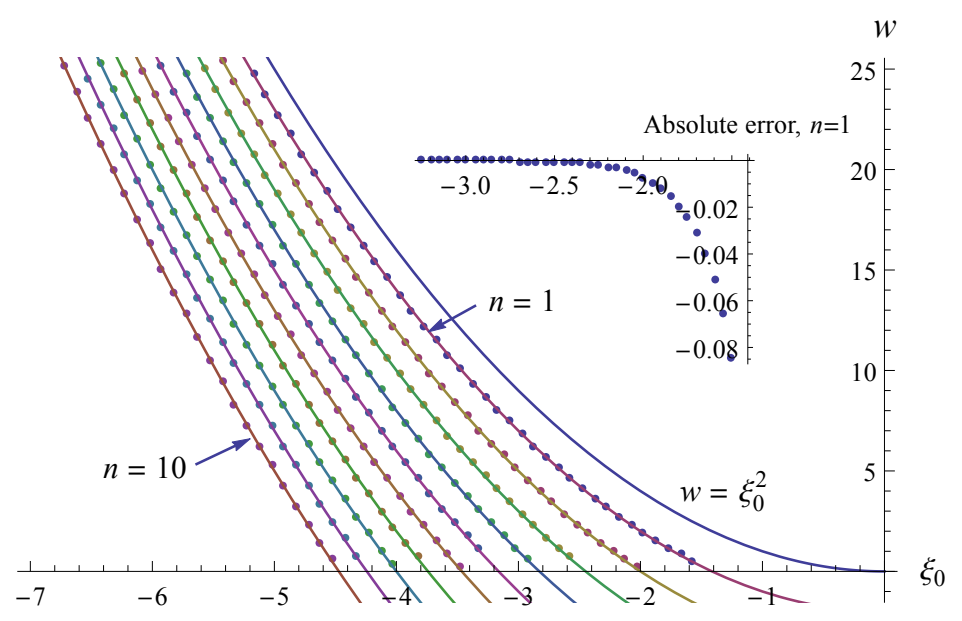

FIG. 3. Approximation (71) (solid curves) with the result of numerical solution of equation (70) (points). The inset shows the absolute error for $n=1$. 
The solution of equation (70) for negative energies (plus sign in (68) and, hence, minus sign in (70) is more complicated (see figure 4). As in the previous case, all the curves belong to the second quadrant. This time the curves can be approximated as

$$
w \approx\left(\xi_{0}^{2}-2 n+a\right)+\Delta
$$

where $a=1 / 3$ and $\Delta=\Delta\left(n, \xi_{0}\right)$ is a correction which starts from zero if $w=0$ and goes to $2-a$ when $\xi_{0} \rightarrow-\infty$. For an insight, an approximation for this correction is given as

$$
\Delta=(2-a) \tanh \left[-2^{1 / 2}\left(\xi_{0}+(2 n-a)^{1 / 2}\right)\right] .
$$

The origin and the structure of this approximation can be revealed if one examines the solution of equation 70 for $w=0$. The latter equation reads

$$
H_{\xi_{0}^{2} / 2}\left(\xi_{0}\right)-\xi_{0} H_{\xi_{0}^{2} / 2-1}\left(\xi_{0}\right)=0
$$

Here, the indexes $\nu=\xi_{0}^{2} / 2$ and $\nu=\xi_{0}^{2} / 2-1$ of the involved Hermite functions are such that they belong to so-called "left transient" region for which $\xi_{0} \approx(2 v+1)^{1 / 2}$ [17] (we recall that $\xi_{0}$ is negative and less than minus one - see Figure 4). Applying then the Airy-function approximation for the Hermite function for this region [32], we find that equation (74) is well approximated by the equation

$$
\sin \left[\pi\left(\frac{\xi_{0}^{2}}{2}+\frac{1}{6}\right)\right]+\frac{\Gamma(7 / 6)}{4 \pi^{1 / 2} 3^{1 / 3}\left(\xi_{0}^{2}\right)^{2 / 3}} \cos \left[\pi\left(\frac{\xi_{0}^{2}}{2}+\frac{1}{6}\right)\right] \approx 0 .
$$

The second term here is proportional to $0.09 /\left|\xi_{0}\right|^{4 / 3}$, hence, it is small for $\left|\xi_{0}\right|>1$. Omitting this term, we arrive at an accurate approximate solution of equation (74) given by

$$
\xi_{0}+(2 n-a)^{1 / 2} \approx 0, n=1,2,3, \ldots
$$

This solution elucidates the structure of approximation 72 , (73): it meets this solution for $w$ close to zero and tends to $w=\xi_{0}^{2}-2(n-1)$ for $\xi_{0} \rightarrow-\infty($ compare with $(71))$.

\section{DISCUSSION}

The sextic oscillator is a quantum mechanical potential that can be discussed in terms of various theoretical approaches. The traditional approach was that based on the quasi-exactly solvable formalism, in which case the solutions for the lowest-lying levels can be expressed 


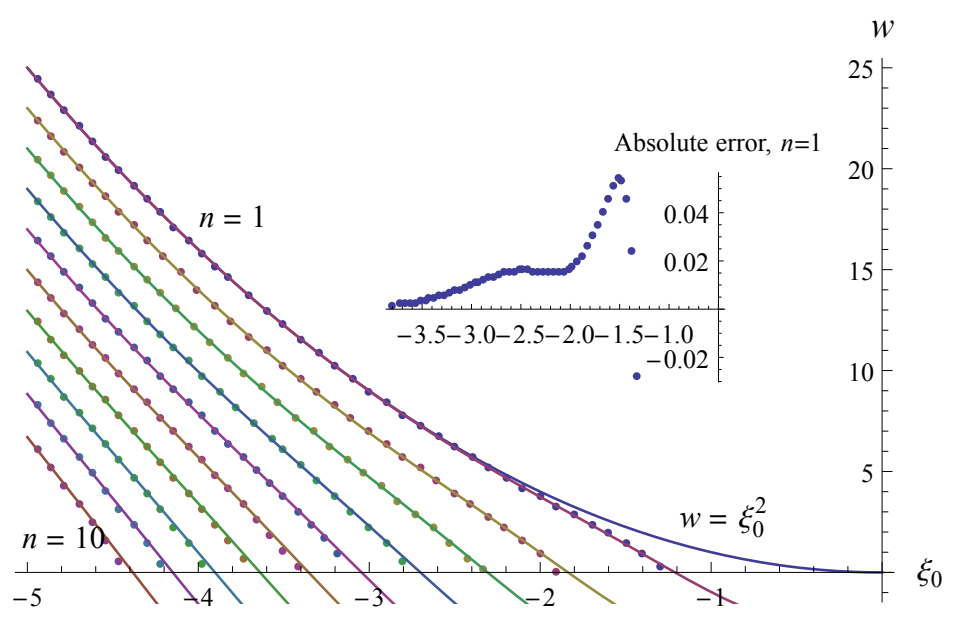

FIG. 4. Approximation (72), (73) (solid curves) compared with the numerical solution of equation (70) (points).

in terms polynomials. More recently it was found this potential can also be discussed by transforming the radial Schrödinger equation into the bi-confluent Heun equation. The solutions in this framework are not necessarily polynomials, so the question how the two approaches are related to each other emerges natually.

We expanded the solutions of the bi-confluent Heun equation in terms of Hermite functions, which can easily be reduced to Hermite polynomials, giving rise to polynomial solutions. In a previous study this connection was proven for the reduced sextic oscillator, i.e. in the absence of the quartic term [24]. Here the general case was studied. The transformation of the Schrödinger equation into the BHE with the solutions expanded in terms of Hermite functions resulted in a three-term recurrence relation for the linear combination coefficients. This recurrence relation could be terminated under certain conditions concerning the potential parameters. In general, the energy eigenvalues then could be determined from a transcendental equation involving a formula containing two adjacent Hermite functions.

One of the possibilities $\left(\nu_{0}=0\right)$ resulted in polynomial solutions, leading directly to the corresponding solutions in terms of the QES framework. The other possibility $\left(\nu_{0}=\gamma-\alpha / \varepsilon\right)$, however, resulted in a non-polynomial solution in general. It was shown that these solutions can also be expressed in terms of a power series in terms of $r^{2}$. At the same time, it turned out that in this approach the $\gamma$ parameter is restricted to non-negative integer values, and this restriction imples that the coupling coefficient appearing in the centrifugal term corresponds 
formally to half-integer vales of the angular momentum $l$. For low values of $N$ the energy eigenvalues can, again, be determined from an algebraic equation of $N+1$ degree. These solutions can also be reduced to those obtained from the QES approach by selecting certain values of the potential parameters.

It has to be mentioned that in the QES approach the coefficient of the quadratic term of the sextic oscillator potential depends on a specific combination $s+M$ of the degree of polynomial appearing in the solution $(M)$ and the parameter appearing in the centrifugal term $(s)$ (see Eq. (42)). This correlation also appears in the case of the BHE approach: see $\alpha$ in Eq. (6). However, in this case setting a fixed value of $V_{2}$ and chosing the possible values of $\gamma$, the appropriate values of $\alpha$ are obtained spontaneously.

The present results indicate the importance of studying the same quantum mechanical potentials in terms of different mathematical approaches (i.e. the QES and the BHE framework in the present case), as they may reveal different aspects of the same exactly solvable problems, and in general, of integrable systems.

\section{ACKNOWLEDGMENTS}

This work was supported by the Science Committee of the Ministry of Education and Science of the Republic of Armenia (SC Grants No. 18RF-139 and No. 18T-1C276), and the Russian-Armenian (Slavonic) University at the expense of the Ministry of Education and Science of the Russian Federation, and the Hungarian Scientific Research Fund - OTKA (Grant No. K112962).

[1] G. A. Natanzon, Vest. Leningrad Univ. 10 (1971) 22; Teor. Mat. Fiz. 38 (1979) 146.

[2] G. Lévai, Int. J. Theor. Phys. 54 (2015) 2724.

[3] A. Ishkhanyan and V. Krainov, Eur. Phys. J. Plus 131 (2016) 342.

[4] C. M. Bender et al., PT Symmetry in Quantum and Classical Mechanics (World Scientific Publishing Europe Ltd., London, 2018).

[5] A.G. Ushveridze, Quasi-exactly solvable models in quantum mechanics (Institute of Physics Publishing, Bristol, 1994).

[6] A. Ronveaux (ed.), Heun's Differential Equations (Oxford University Press, London, 1995). 
[7] S.Yu. Slavyanov and W. Lay, Special functions (Oxford University Press, Oxford, 2000).

[8] F. W. J. Olver, D.W. Lozier, R.F. Boisvert, and C.W. Clark (eds.), NIST Handbook of Mathematical Functions (Cambridge University Press, New York, 2010).

[9] A. Lemieux and A.K. Bose, Ann. Inst. Henri Poincaré 10 (1969) 259.

[10] D. Batic, R. Williams, M. Nowakowski, J. Phys. A 46 (2013) 245204.

[11] A. Ishkhanyan, Ann. Phys. (N. Y.) 388 (2018) 456.

[12] D. Batic, D. Mills-Howell, M. Nowakowski, J. Phys. A 56 (2015) 052106.

[13] A. M. Ishkhanyan, Theor. Math. Phys. 188 (2016) 980.

[14] T.A. Ishkhanyan and A.M. Ishkhanyan, Ann. Phys. 383 (2017) 79.

[15] A. Erdélyi, Q. J. Math. (Oxford) 15 (1944) 62.

[16] D. Schmidt, J. Reine Angew. Math. 309 (1979) 127.

[17] L. J. El-Jaick and B. D. B. Figueiredo, J. Math. Phys. 50 (2009) 123511.

[18] A. López-Ortega, Phys. Scr. 90 (2016) 085202.

[19] A. M. Ishkhanyan, EPL 112 (2015) 10006.

[20] A. M. Ishkhanyan, Mod. Phys. Lett. A 31 (2016) 1650177.

[21] A. M. Ishkhanyan, Phys. Lett. A 380 (2016) 3786.

[22] A. López-Ortega, arXiv:1512.04196 [math-ph] (2015).

[23] A. M. Ishkhanyan, Eur. Phys. Lett. 115 (2016) 20002.

[24] G. Lévai and A. M. Ishkhanyan, Mod. Phys. Lett. A 31 (2016) 1650177.

[25] A. V. Turbiner and A. G. Ushveridze, Phys. Lett. A 126 (1987) 181.

[26] A. Turbiner, Commun. Math. Phys. 118 (1988) 467.

[27] C. M. Bender and G. V. Dunne, J. Math. Phys. 37 (1996) 6.

[28] N. Saad, R. L. Hall and H. Çiftçi, J. Phys. A 39 (2006) 8477-8486.

[29] G. Lévai and J. M. Arias, Phys. Rev. C 69 (2004) 014304

[30] G. Lévai and J. M. Arias, Phys. Rev. C 81 (2010) 044304

[31] R. Budaca, P. Buganu, M. Chabab, A. Lahbas and M. Oulne, Ann. Phys. 375 (2016) 65.

[32] G. Szegő, Orthogonal polynomials, Amer. Math. Soc. Colloquium Publications 23, (1939) 344. 